\title{
Is there a relationship between sleep and obesity?
}

\section{Opinion}

Yes. Several epidemiological and experimental evidences confirm the association between decreased hours of sleep and increased risk of obesity.

Sleep is an important modulator of neuroendocrine function and glucose metabolism. Reduced bedtime results in metabolic and endocrine changes, including decreased glucose tolerance and insulin sensitivity, imbalance of anorectic and orexigen hormones, and consequently increased appetite.

Epidemiological studies, conducted in different geographic regions, have examined the association between sleep and obesity in both adults and children. Most studies found a significant association between short sleep ( $<6$ hours per night) and increased risk for obesity. A meta-analysis of 18 studies with 604,509 adult subjects demonstrated an increased relative risk for obesity of $1.55(\mathrm{p}<0.0001)$ for those who slept less than 5 hours of sleep.

Buxton \& Marcelli ${ }^{1}$ conducted a study with 56,507 adults between 18 and 85 years of age who demonstrated a $6 \%$ increase in the probability of developing obesity in subjects who had sleep (selfreported) of less than 7 hours per night.

The mechanisms related to sleeping hours and obesity are not yet fully understood, but the researchers point out that the disturbances caused by the decrease in sleeping hours influence appetite, satiety and, consequently, food intake, favoring an increase in obesity.

Studies have shown that ghrelin, an orexigenic hormone (which promotes hunger) is increased after the restriction of sleeping hours, while leptin, an anorexigenic hormone (which contributes to the perception of satiety), is diminished. In addition, the reduction of hours of sleep can affect body energy expenditure, since leptin is a hormone that increases energy expenditure and, therefore, changes in leptin levels after sleep deprivation affect both caloric intake and expenditure energy.

\author{
Volume 6 Issue 4 - 2018 \\ Carolina Mantelli Borges \\ Medical Endocrinologist and Metabologist, Brazil \\ Correspondence: Carolina Mantelli Borges, Medical \\ Endocrinologist and Metabologist, Brazil, \\ Email dramantelli@hotmail.com \\ Received: June 26, 2018 | Published: July 23, 2018
}

According to the authors of these studies, sleep is a restorative process of the brain, important for physical and mental health. Decreased sleep duration is quite common in society and raises concerns about the negative impact of sleep disorders on overall health.

\section{Acknowledgments}

None.

\section{Conflict of interest}

The author declares there is no conflict of interest.

\section{References}

1. Buxton OM, Marcelli E. Short and long sleep are positively associated with obesity, diabetes, hypertension, and cardiovascular disease among adults in the United States. Soc Sci Med. 2010;71(5):1027-1036. 